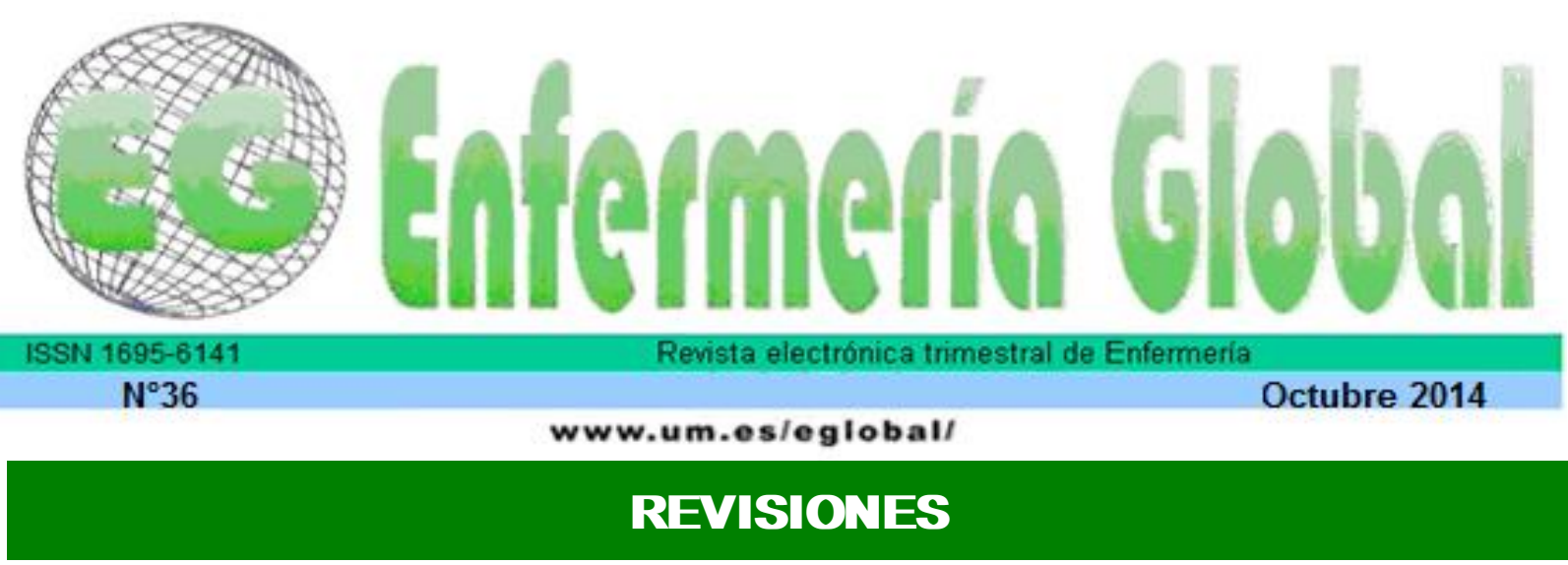

\title{
Enfermedad de Crohn, cronicidad y rol enfermero: revisión integradora y estado de la cuestión
}

Crohn's disease, chronic and nurse role: literature review and state of affairs

\author{
*García Sanjuan, Sofía *Lillo Crespo, Manuel *Sanjuan Quiles, Ángela \\ *Richart Martínez, Miguel \\ *Departamento de Enfermería. Facultad Ciencias de la Salud. Universidad Alicante. E-mail:
$\frac{\text { sofia.garcia@ua.es }}{\text { (Esta investigación es apoyada por la Beca VALI + D (ACIF/2013/141García-SanjuánSofía). }}$
Consellería de Educación, Cultura y Deporte. Generalitat Valenciana. España).
}

Palabras clave: Enfermedad crónica; experiencia de vida; enfermedad de Crohn; enfermedad inflamatoria intestinal; enfermería; enfermera.

Keywords: Chronic illness; experience of living; Crohn' s disease and inflammatory bowel disease; nurse, nursing

\section{RESUMEN}

Objetivo: Conocer las experiencias de vida y la percepción del apoyo social en las personas afectadas por la Enfermedad de Crohn (EC), así como el rol que desempeñan las enfermeras/os en dicho proceso.

Método: La búsqueda se realizó en PUBMED, CUIDEN, CINHAL, CSIC, SCIELO, TESEO. También se utilizó la búsqueda sensible, REDICS (Red de investigación cualitativa en salud) y RUA (Repositorio Institucional de la Universidad de Alicante).

Resultados: Tras aplicar los criterios de exclusión, se identificaron 20 artículos que por su título y resumen se consideraron pertinentes para un examen más detallado (texto completo). Las posibles discrepancias se resolvieron por consenso entre el investigador principal y dos investigadores más del estudio. La revisión se llevó a cabo entre enero de 2011 y diciembre de 2011.

Conclusiones: Son pocos los estudios que se ocupan de conocer la experiencia de vivir con la EC. La poca literatura recuperada que se encarga de estos temas muestra las luchas y afrontamientos que realizan los afectados. La enfermera/o suele ser la persona de referencia como único apoyo para manejar el afrontamiento de dicha enfermedad. Las funciones de la enfermera/o como apoyo de los afectados, muestran un rol amplio que puede cubrir todas las necesidades de las personas con EC. 


\section{ABSTRACT}

Objective: to know the experiences of life and perception of social support for people affected by Crohn's disease (CD), and the role played by nurses in the process.

Methods: The PubMed search was conducted, CUIDEN, CINAHL, CSIC, SCIELO, Theseus. Also used sensitive search, REDICS (Red qualitative health research) and RUA (Institutional Repository of the University of Alicante).

Results: After applying the exclusion criteria, 20 articles were identified by title and summary were considered relevant for further scrutiny (full text). The discrepancies were resolved by consensus between the principal investigator and two researchers of the study. The review was carried out between January 2011 and December 2011.

Conclusions: there are few studies that deal with knowing the experience of living with the EC. The recovered little literature that deals with these issues shows the struggles and confrontations that perform those affected. The nurse / or the person is often referred to as sole support to handle coping with the disease. The functions of the nurse / or support of those affected, showing a role can I expand to cover all the needs of people with CD.

\section{INTRODUCCIÓN, JUSTIFICACIÓN Y OBJETIVOS}

La bibliografía es rica en información clínica acerca de la Enfermedad de Crohn (EC) y nos habla de una enfermedad crónica asociada a una inflamación gastrointestinal de etiopatogenia desconocida. Debido a su carácter autoinmune y su comienzo insidioso su diagnóstico puede retrasarse a lo largo del tiempo ${ }^{(1-3)}$.

Los síntomas de la EC son fluctuantes e impredecibles, los más característicos son diarrea crónica, hemorragia rectal, dolor abdominal con retortijones, fiebre, alternancia de cuadros de defecación normal con diarreicos, pérdida de apetito, pérdida de peso, náuseas y vómitos, con períodos de remisión y recaídas, y en muchos casos, llegan a requerir hospitalizaciones con asiduidad e intervenciones quirúrgicas que pueden desembocar en ostomías ${ }^{(4)}$ con las implicaciones psicológicas y sociales que conlleva para el individuo, del tipo de: trastorno de imagen, desvalorización, ruptura con su modo de vida, depresión... ${ }^{(5,6)}$

Consecuentemente, las personas afectadas por la enfermedad, no sólo sufren los síntomas derivados de la propia enfermedad tal y como nos lo indica la bibliografía clínica publicada, sino que además cuando se encuentran en periodos de remisión, deben hacer frente a numerosos efectos secundarios provocados por los tratamientos que en la actualidad se instauran en este tipo de patologías, como corticoides y terapias biológicas ${ }^{(7-10)}$.

Realizado el análisis de la bibliografía recuperada, se parte del siguiente supuesto que ha servido como hipótesis, guía e impulso para llevar a cabo este trabajo: tras saberse conocedor del diagnóstico de EC, la persona tiene que replantearse la forma de vida, ya que además de ser portador de la etiqueta de paciente crónico, dicha nueva condición puede limitar la actividad física, hábitos alimenticios y actividades que estén lejos de un cuarto de baño.

Llegados a este punto, se hace necesario aclarar que el propósito de esta revisión no es realizar un estudio en profundidad sobre todo lo que se sabe acerca de los signos y síntomas de la enfermedad, como aportan las evidencias citadas anteriormente. El objetivo de este trabajo es analizar a través de una revisión integradora qué sentimientos, emociones, adaptaciones y cambios en los estilos de vida suceden en 
las personas desde que conocen el diagnóstico de EC, su afrontamiento, el manejo que hacen de su propia enfermedad, los cambios sufridos en su cotidianeidad y el alcance de las funciones desarrolladas por enfermería para convertirse en un elemento más de la red de apoyo de las personas afectadas con estas patologías en diferentes partes del mundo.

\section{Estrategia de búsqueda}

Las bases de datos utilizadas fueron: PUBMED, CUIDEN, CINHAL, CSIC, SCIELO, TESEO. También se utilizó la búsqueda sensible, REDICS (Red de investigación cualitativa en salud) y RUA (Repositorio Institucional de la Universidad de Alicante).

Se definieron las palabras clave, tanto Desc o tesauros como lenguaje natural: Chronic illness, life experience, Crohn's disease, bowel inflammatory disease, nurse, nursing, enfermedad crónica, experiencia de vida, enfermedad de Crohn, enfermedad inflamatoria intestinal, enfermería, enfermera y se combinaron a través de los operadores booleanos "OR" y "AND" ("o", "y").

Para la inclusión de los diferentes trabajos recuperados en esta revisión bibliográfica, se limitó el idioma a inglés, portugués y español, publicaciones comprendidas entre enero de 2000 y diciembre de 2011, lógicamente con la actitud de no excluir posibles trabajos o estudios que aún siendo más antiguos puedan seguir siendo un referente en la materia, estudios de diseño tanto cuantitativo como cualitativo, literatura gris, también denominada como literatura no convencional, literatura semipublicada o literatura invisible por tratarse de cualquier tipo de documento que no se difunde por los canales ordinarios de publicación comercial, y que por tanto plantea problemas de acceso incluyendo las investigaciones en curso, que se utilizaron para complementar los trabajos publicados, que cumplieran al menos dos de las categorías propuestas en el eje central del estudio "Cronicidad, enfermería y enfermedad de Crohn" y que estuvieran formulados en relación al tema a investigar.

\section{RESULTADOS}

Las referencias recuperadas fueron revisadas en su totalidad de forma independiente, las posibles discrepancias se resolvieron por consenso entre el investigador principal y dos investigadores más del estudio. La revisión se llevó a cabo entre enero de 2011 y diciembre de 2011, rechazando aquellos que no cumplían los criterios de inclusión propuestos. Tras la primera búsqueda se recuperaron 1875 artículos, retirando las referencias cruzadas en las diferentes bases de datos. Con el análisis del resumen se identificaron 97 artículos, tras la lectura completa fueron seleccionados e incluidos en esta revisión 20 artículos Fig 1. 
Fig. 1 Diagrama donde se muestra el proceso de identificación y selección de estudios

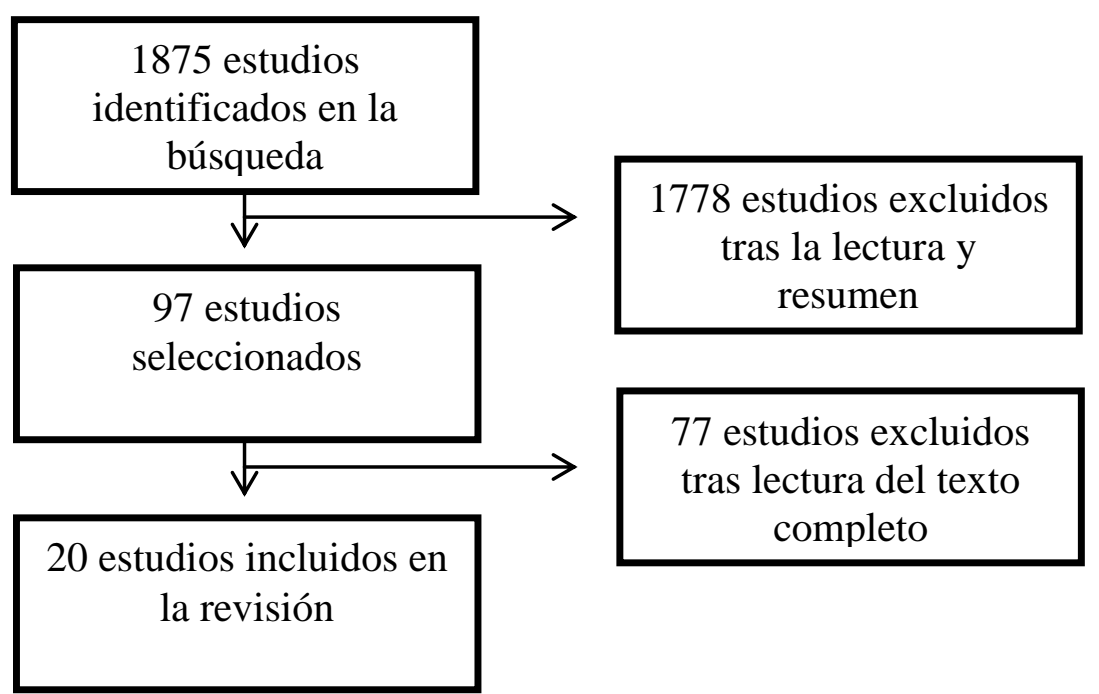

El rigor metodológico de la literatura recuperada se tuvo en cuenta al revisar los resultados. De hecho, la diversa naturaleza de los estudios (diferentes tamaños en la muestra, diferentes diseños, criterios de calidad...) hacen que cualquier generalización de las conclusiones deba tratarse con cautela. Un resumen de las características de los estudios incluidos aparece en la Fig. 2

Fig. 2 Características de los estudios incluidos

\begin{tabular}{|c|c|c|c|c|c|}
\hline Autores & Título & Publicación & Año & País & $\begin{array}{l}\text { Categorías eje } \\
\text { central estudio }\end{array}$ \\
\hline Medina $\mathrm{O}$. & Encuentro con la cronicidad & $\begin{array}{l}\text { Rev } \\
\text { Desarrollo } \\
\text { Cientif } \\
\text { Enferm }\end{array}$ & 2003 & España & $\begin{array}{l}\text { Cronicidad y } \\
\text { enfermería }\end{array}$ \\
\hline $\begin{array}{l}\text { Muñoz LA, Price } \\
\text { Y, Gambini L, } \\
\text { Stanfanelli M } \\
\end{array}$ & $\begin{array}{l}\text { Significados simbólicos de los } \\
\text { pacientes con enfermedades } \\
\text { crónicas }\end{array}$ & $\begin{array}{l}\text { Rev Esc } \\
\text { Enferm USP }\end{array}$ & 2003 & Brasil & $\begin{array}{l}\text { Cronicidad y } \\
\text { enfermería }\end{array}$ \\
\hline $\begin{array}{l}\text { Cònsul M, } \\
\text { Soldevilla M }\end{array}$ & $\begin{array}{l}\text { La enfermedad inflamatoria } \\
\text { intestinal. Reflexiones sobre los } \\
\text { cuidados enfermeros }\end{array}$ & Nursing & 2004 & España & $\begin{array}{l}\text { Cronicidad, } \\
\text { enfermería y EC }\end{array}$ \\
\hline Barrnett M & $\begin{array}{l}\text { Chronic obstructive pulmonary } \\
\text { disease: A phenomenological } \\
\text { study of patient's experience }\end{array}$ & $\begin{array}{l}\text { Journal of } \\
\text { Clinical } \\
\text { Nursing } \\
\end{array}$ & 2005 & Inglaterra & $\begin{array}{l}\text { Cronicidad y } \\
\text { enfermería }\end{array}$ \\
\hline $\begin{array}{l}\text { Woods L, Belling } \\
\text { R, McLaren S }\end{array}$ & $\begin{array}{l}\text { A systematic review of the } \\
\text { effectiveness of inflammatory } \\
\text { bowel disease specialist nurse }\end{array}$ & $\begin{array}{l}\text { London South } \\
\text { Bank } \\
\text { University }\end{array}$ & 2006 & $\begin{array}{l}\text { Reino } \\
\text { Unido }\end{array}$ & $\begin{array}{l}\text { Cronicidad, } \\
\text { enfermería y EC }\end{array}$ \\
\hline Ibarra X, Siles J. & $\begin{array}{l}\text { Rol del enfermo crónico: Una } \\
\text { revisión crítica desde la } \\
\text { perspectiva de los cuidados } \\
\text { enfermeros. }\end{array}$ & $\begin{array}{l}\text { Cultura de los } \\
\text { Cuidados }\end{array}$ & 2006 & España & $\begin{array}{l}\text { Cronicidad y } \\
\text { enfermería }\end{array}$ \\
\hline Pont E & Visiones desde la enfermedad & $\begin{array}{l}\text { Metas de } \\
\text { Enfermería }\end{array}$ & 2006 & España & $\begin{array}{l}\text { Cronicidad y } \\
\text { enfermería }\end{array}$ \\
\hline $\begin{array}{l}\text { Mercado FJ, Ibarra } \\
\text { E }\end{array}$ & $\begin{array}{l}\text { Chronic illness from the } \\
\text { perspective of patiens and } \\
\text { health professionals: A } \\
\text { qualitative study in Mexico }\end{array}$ & $\begin{array}{l}\text { Cadernos De } \\
\text { Saúde Pública }\end{array}$ & 2007 & México & $\begin{array}{l}\text { Cronicidad y } \\
\text { enfermería }\end{array}$ \\
\hline
\end{tabular}




\begin{tabular}{|c|c|c|c|c|c|}
\hline $\begin{array}{l}\text { Tobón S, Vinaccia } \\
\text { S, Quiceno JM, } \\
\text { Sandín B, Rojas } \\
\text { ACN }\end{array}$ & $\begin{array}{l}\text { Aspectos psicopatológicos en la } \\
\text { enfermedad de Crohn y en la } \\
\text { colitis ulcerosa }\end{array}$ & $\begin{array}{l}\text { Avances en } \\
\text { Psicología } \\
\text { Latinoamerica } \\
\text { na }\end{array}$ & 2007 & Colombia & Cronicidad y EC \\
\hline $\begin{array}{l}\text { Stanfield C, Frase } \\
\text { A, Thomson J, } \\
\text { Povey J }\end{array}$ & $\begin{array}{l}\text { Roles descriptive for } \\
\text { inflammatory bowel disease } \\
\text { nurse specialists }\end{array}$ & $\begin{array}{l}\text { Royal College } \\
\text { of Nursing }\end{array}$ & 2007 & $\begin{array}{l}\text { Reino } \\
\text { Unido }\end{array}$ & $\begin{array}{l}\text { Cronicidad, } \\
\text { Enfermería y } \\
\text { EC }\end{array}$ \\
\hline Michael WD & $\begin{array}{l}\text { Fight back against inflammatory } \\
\text { Bowel disease }\end{array}$ & Nursing & 2008 & USA & $\begin{array}{l}\text { Cronicidad, } \\
\text { enfermería y } \\
\text { EC }\end{array}$ \\
\hline $\begin{array}{l}\text { Belling R, Woods } \\
\text { L, MacLaren S. }\end{array}$ & $\begin{array}{l}\text { Stakecholder perceptions of } \\
\text { specialist Inflammatory Bowel } \\
\text { Disease nurse's role and } \\
\text { personal attributes }\end{array}$ & $\begin{array}{l}\text { International } \\
\text { Journal of } \\
\text { Nursing } \\
\text { Practice }\end{array}$ & 2008 & $\begin{array}{l}\text { Reino } \\
\text { Unido }\end{array}$ & $\begin{array}{l}\text { Cronicidad, } \\
\text { enfermería y EC }\end{array}$ \\
\hline Moreira MC & $\begin{array}{l}\text { Colitis Ulcerosa \& Enfermedad } \\
\text { de Crohn }\end{array}$ & $\begin{array}{l}\text { Universidad } \\
\text { de Barcelona }\end{array}$ & 2009 & España & Cronicidad y EC \\
\hline
\end{tabular}

\begin{tabular}{|c|c|c|c|c|c|}
\hline $\begin{array}{l}\text { Iglesias M, } \\
\text { Barriero de Acosta } \\
\text { M, Vázquez I, } \\
\text { Figueiras A, Nieto } \\
\text { L, Lorenzo A, et al }\end{array}$ & $\begin{array}{l}\text { Impacto psicológico en la } \\
\text { enfermedad de Crohn en } \\
\text { pacientes en remisión: Riesgo } \\
\text { de ansiedad y depresión }\end{array}$ & $\begin{array}{l}\text { Rev Esp } \\
\text { Enferm Dig }\end{array}$ & 2009 & España & Cronicidad y EC \\
\hline $\begin{array}{l}\text { Fernández JC, } \\
\text { Simón MA, Bueno } \\
\text { AM }\end{array}$ & $\begin{array}{l}\text { Depresión, género y } \\
\text { manifestaciones sistémicas en } \\
\text { las enfermedades inflamatorias } \\
\text { crónicas del intestino. }\end{array}$ & Psicothema & 2010 & España & Cronicidad y EC \\
\hline Autores & Título & Publicación & Año & País & $\begin{array}{l}\text { Categorías eje } \\
\text { central estudio }\end{array}$ \\
\hline $\begin{array}{l}\text { Hernández- } \\
\text { Sanpelayo P, } \\
\text { Seoane M, Oltra L, } \\
\text { Marín L, Torrejón } \\
\text { A, Vera MI, et al }\end{array}$ & $\begin{array}{l}\text { Contribution Of nurses to the } \\
\text { quality of care in management } \\
\text { of inflammatory Bowel disease: } \\
\text { A synthesis of the evidence. }\end{array}$ & $\begin{array}{l}\text { Journal of } \\
\text { Crohn's an } \\
\text { Colitis }\end{array}$ & 2010 & $\begin{array}{l}\text { Reino } \\
\text { Unido }\end{array}$ & $\begin{array}{l}\text { Cronicidad, } \\
\text { Enfermería y } \\
\text { EC }\end{array}$ \\
\hline $\begin{array}{l}\text { Cooper JM, Cilier } \\
\text { J, James V, } \\
\text { Hawkey JC }\end{array}$ & $\begin{array}{l}\text { Beliefs about personal control } \\
\text { and self-management in } 30-40 \\
\text { years olds living with } \\
\text { inflammatory bowel disease: A } \\
\text { qualitative study }\end{array}$ & $\begin{array}{l}\text { Int J Nurs } \\
\text { Stud }\end{array}$ & 2010 & USA & $\begin{array}{l}\text { Cronicidad, } \\
\text { enfermería y EC }\end{array}$ \\
\hline $\begin{array}{l}\text { Reza C, Castro M, } \\
\text { Solano G, Torres } \\
\text { D, Martínez V }\end{array}$ & $\begin{array}{l}\text { El impacto del diagnóstico. } \\
\text { Experiencia descrita por } \\
\text { mujeres con hipertensión } \\
\text { arterial }\end{array}$ & $\begin{array}{l}\text { Esc Anna } \\
\text { Nery Rev } \\
\text { Enfer }\end{array}$ & 2010 & México & $\begin{array}{l}\text { Cronicidad y } \\
\text { enfermería }\end{array}$ \\
\hline Diener JA & $\begin{array}{l}\text { Everyone poops but no one } \\
\text { wants to talk about it: The lived } \\
\text { experiences of Young people } \\
\text { with infammatory Bowel } \\
\text { disease }\end{array}$ & $\begin{array}{l}\text { Universidad } \\
\text { de Canadá } \\
\text { [Tesis } \\
\text { doctoral] }\end{array}$ & 2011 & Canadá & $\begin{array}{l}\text { Cronicidad, } \\
\text { enfermería y EC }\end{array}$ \\
\hline $\begin{array}{l}\text { Casellas F, Borruel } \\
\text { N, Torrejón A, } \\
\text { Castells I }\end{array}$ & $\begin{array}{l}\text { Efecto de la aplicación de un } \\
\text { método de asistencia continuada } \\
\text { centrada en el paciente con } \\
\text { enfermedad inflamatoria } \\
\text { intestinal en la actividad } \\
\text { hospitalaria }\end{array}$ & $\begin{array}{l}\text { Rev Esp } \\
\text { Enferm Dig }\end{array}$ & 2012 & España & $\begin{array}{l}\text { Cronicidad, } \\
\text { enfermería y EC }\end{array}$ \\
\hline
\end{tabular}

\section{Desarrollo y Análisis de datos}

En el análisis de los hallazgos recopilados no se ha tratado de forma aislada y exclusiva aquella información acerca de la EC (lo cual podría haber desembocado en 
un análisis o estado de la cuestión descontextualizado) sino que se ha empleado un modelo de análisis basado en 3 categorías que constituyen el eje central del estado de la cuestión Fig. 3. Se entiende que cada uno de los conceptos que se muestran en esta figura son mucho más amplios, de hecho, la EC también es susceptible de un análisis desde la perspectiva clínica (haciendo hincapié en signos y síntomas), la cronicidad podría ser analizada desde la vivencia de otras patologías crónicas y el apoyo enfermero podría ser descrito en diferentes contextos y situaciones de salud y enfermedad de los individuos; sin embargo, se ha buscado sintetizar mediante la revisión integradora: la vivencia de la cronicidad, desde la perspectiva de la EC y con el apoyo de la enfermera/o para este caso concreto y que coincide con el eje central de este estudio. cuestión

Fig. 3 Tres categorías que forman el eje central del estudio o estado de la

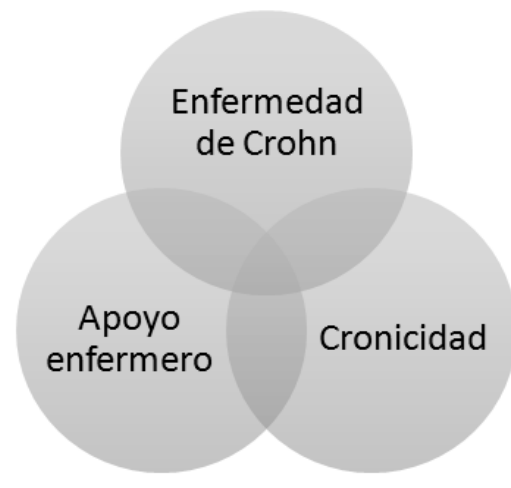

Para analizar el concepto de cronicidad se parte de la siguiente definición, una enfermedad crónica es aquella en la que los síntomas de la persona se prolongan a largo plazo de manera que perjudican su capacidad para seguir con actividades significativas y rutinas normales. Su tratamiento médico suele tener una eficacia limitada y contribuye a veces, al malestar físico y psicológico de las personas y sus familias ${ }^{(11)}$. Es importante que los profesionales de la salud, tengan conciencia del impacto que tiene su diagnóstico, tanto en el individuo como en su entorno, ya que muchos de ellos buscarán ayuda en diferentes colectivos profesionales, tanto para aliviar sus síntomas como sus aflicciones psicológicas.

El diagnóstico de una enfermedad crónica es el resultado de un complejo entramado de interacciones. El proceso puede durar años y es posible que no se resuelva hasta la muerte y la autopsia. Desde el diagnóstico, la manera de ver, oír y reflexionar sobre las cosas parece cambiar. "Es doloroso afrontar de lleno las implicaciones del hecho de que las realidades establecidas de la propia vida diaria se vuelvan repentinamente inestables, la enfermedad crónica desbarata todo lo que damos por supuesto" (12).

Son varios los autores que afirman que los individuos con una enfermedad crónica experimentan con frecuencia una imagen deteriorada de sí mismos y no son capaces de asumir una nueva que tenga el mismo valor que la anterior. Incluso pueden llegar a sentir que sus vidas tienen menor valor que las demás y sentirse una carga para su entorno, con la disminución de autoestima que esto supone ${ }^{(13-14)}$.

Según Charmaz ${ }^{(15)}$ con el mantenimiento de una cronicidad se alteran aspectos del "yo" que la persona ya tenía establecidos como base de su propia identidad. Su vida comienza a girar en torno a su propia enfermedad, apareciendo una serie de 
restricciones que van a modificar su vida. El mantenimiento de una cronicidad, lleva a la persona a perder la libertad, la identidad, a un descenso de la autoestima y por lo tanto a una devaluación de la propia persona.

Se realizó un estudio en 2009 en la ciudad de México ${ }^{(16)}$, con el fin de conocer la experiencia de mujeres en el momento de recibir un diagnóstico de cronicidad. Los resultados de dicho estudio revelaron que el individuo atraviesa varias etapas, a saber, en un primer momento pasa de la alegría a la angustia, llegando incluso a la depresión, reconociendo un proceso de lucha-adaptación, que en muchas ocasiones les lleva a manifestar trastornos emocionales o procesos de descompensación, lo que impide el control de la propia enfermedad. Por último, el individuo intenta buscar estrategias para enfrentar la propia cronicidad, con el deseo de prolongar la vida de tal forma que la recuperación permita conservar la independencia.

En relación al proceso de adaptación y a las etapas que atraviesa una persona en el momento del diagnóstico de cronicidad, de acuerdo con Kübler-Ross (17), la adaptación es similar a sus conocidas etapas sobre el duelo. La primera reacción emocional al tener conocimiento de que se padece una enfermedad crónica es la negación "yo no tengo eso", ya que cuesta aceptar una realidad que no queremos. La ira llega cuando ya se ha comprobado sin duda alguna la enfermedad, “por qué yo?”, "yo no merezco esto". El individuo está descargando sentimientos de hostilidad, siente y padece la injusticia de su situación. Este sentimiento se convierte en depresión cuando la persona reconoce la inevitable realidad e incluso el enfermo hace un último intento de llegar a un compromiso con la realidad, confabulando negociaciones con su propio yo. Y finalmente, y sólo habiendo pasado por las cuatro etapas anteriores, la persona acepta su enfermedad como parte de su yo, una realidad con la que debe vivir.

El saberse portador de una enfermedad crónica, pone de manifiesto que esta nueva situación abre puertas de enfrentamiento a sentimientos como la fragilidad, la soledad y el miedo, como explica el doctor Pont (18) "El miedo brota de la incapacidad de concebirnos y aceptarnos vivos en la cárcel de un cuerpo enfermo. La enfermedad crónica es como el despertar abrupto en medio de un bello sueño, una ruptura que puede aterrorizar". Según las palabras de Pont, se podría decir que la enfermedad hace que la persona tenga que enfrentarse a nuevos sentimientos que quizá jamás hubiera experimentado antes y que tendrá que conocerlos, superarlos o aprender a vivir con ellos, ya que a partir del diagnóstico formarán parte de su nueva vida.

En un intento de describir la vivencia y las convergencias de diferentes personas afectadas por diferentes patologías crónicas, Muñoz ${ }^{(19)}$ concluye que los diversos grupos coinciden en las dimensiones de pérdida de bienestar y soporte social, miedo a la invalidez y a la muerte, las formas en las que perciben la familia y en la aceptación de la cronicidad. Por lo que podemos entender que a pesar de que las patologías sean diferentes tanto en signos como en síntomas, hasta incluso en limitaciones o cambios en sus vidas, las personas afectadas por la cronicidad, coinciden en ciertas facetas de cómo va a cambiar su día a día y la percepción del mundo que les rodea.

En palabras de Anota ${ }^{(20)}$ aceptar la enfermedad en el inicio no es tarea fácil para la persona afectada, el diagnóstico de cronicidad lleva consigo un sentimiento de conflicto, de culpa, la ruptura del estilo de vida al que está acostumbrado, la incertidumbre del futuro y el modo como se desarrollará su vida ya que se abandonan 
los proyectos presentes y futuros. Bury ${ }^{(21)}$, por ejemplo, define que en el inicio de la aceptación de la enfermedad se produce una "ruptura biográfica", donde la persona tendrá que reorganizar su vida y poner a prueba su capacidad de aprendizaje y adaptación a su nueva condición. Algunos autores como Barnett, Faria o Mercado ${ }^{(22-}$ 24) afirman que enfermar y enfrentar la enfermedad permite reordenar la forma habitual de vivir, pues cada sujeto tiene experiencias particulares y únicas con su enfermedad, no sólo en su ámbito orgánico o corporal, sino en otros aspectos de su vida laboral, social y emocional. Por lo que es importante conocer las etapas que atraviesa una persona que acaba de recibir un diagnóstico de cronicidad, de una forma única e individual, consiguiendo así, cubrir sus necesidades de una forma integral.

Mucho falta todavía para poder explicar la etiopatogenia e historia natural de la EC, a pesar de que sí se han identificado algunos factores de riesgo, como el tabaco ${ }^{(25-26)}$, la predisposición genética ${ }^{(1)}$, la lactancia artificial ${ }^{(27)}$, la alimentación, infecciones y sobre todo factores emocionales, tanto en la aparición de la enfermedad como durante el curso de la misma, por lo que aún no existe una atención correcta, preventiva y terapéutica hacia las personas que conviven con dicha enfermedad. Se desprende según Vega ${ }^{(28)}$ o Diener ${ }^{(29)}$, que estas personas presentan desajustes psicológicos, como depresión y ansiedad, debidos a las propias características de la enfermedad (cronicidad, interferencia de su vida cotidiana, limitaciones, delegación de responsabilidad, posible cirugía...), condiciones estresantes éstas, suficientes para explicar dichos desajustes.

Una persona afectada por la EC, no sólo debe hacer frente a los síntomas propios de la enfermedad (diarrea, vómitos, dolores abdominales...), sino que también tendrá que asumir las manifestaciones extra digestivas y superar los efectos secundarios de los diferentes tratamientos con los que se trata esta patología (estrías, ansiedad, dependencia, problemas oculares, disminución de la fertilidad...) ${ }^{(4,5,7,10,30)}$.

Cuando las personas con esta sintomatología comienzan su recorrido por las diferentes pruebas para llegar al diagnóstico, experimentan frustración, miedo e incertidumbre, además de la pérdida de control de su propio cuerpo; esa sensación no mejora cuando finalmente son diagnosticados con la EC, ya que se convierten en "objeto" de una gran variedad de pruebas, tratamientos invasivos y medicaciones agresivas que acaban por llevarlos a la pérdida de fe en el sistema sanitario, puesto que en muchas ocasiones la sintomatología no remite ${ }^{(29)}$.

Esta pérdida de esperanza en una recuperación, unida al dolor físico y al sufrimiento, lleva a las personas con EC a un estado de enfado y vulnerabilidad que hace que sean ellos los que tomen, por iniciativa propia, la riendas de su propio tratamiento, y son capaces de probar cualquier "remedio" que pueda hacer mejorar sus síntomas, como modificación de la dieta o la utilización de terapias alternativas, llegando incluso a rechazar los tratamientos convencionales ${ }^{(29)}$.

Una vez que termina su peregrinaje en la búsqueda de cualquier alternativa y comprenden que los tratamientos médicos son importantes para el alivio de los síntomas, pero que a la vez resultan limitados, sólo les queda aprender a vivir con la enfermedad crónica, una enfermedad que como apuntan diferentes estudios (31-35), está salpicada de un cierto estigma social, ya que puede resultar vergonzoso hablar de la sintomatología (meteorismos, diarreas, movimientos intestinales frecuentes, urgencia...) de esta patología en según qué círculos. 
Según Diener ${ }^{(29)}$ la personalidad juega un papel importante en esta enfermedad, ya que está relacionada directamente con la forma de afrontar sucesos vitales. La EC genera sentimientos de baja autoestima y pérdida de libertad, incluso las personas afectadas por la enfermedad, restringirán sus actividades, ya que éstas quedarán limitadas a aquellas en las que haya un baño cerca. Esto supone que la persona tenga que cambiar sus hábitos de vida, incluso que se vea obligado a dejar su trabajo, con la consiguiente devaluación que supone para una persona no poder vivir la vida que había construido antes de saberse enfermo crónico ${ }^{(15)}$.

La EC, es un factor potencial que interfiere en la calidad de vida del sujeto que la padece y que además facilita la aparición de acontecimientos como disconfort durante largos periodos, múltiples hospitalizaciones, procedimientos intrusivos de diagnóstico y pérdida de responsabilidad laboral, familiar, social y de identidad ${ }^{(10,36-39)}$.

El Rol que debe poseer la enfermera/o en la EC comenzará en el mismo momento del diagnóstico, ya que dicho diagnóstico predispone a los pacientes a desarrollar nuevas estrategias para afrontar su situación. Entre estas estrategias de afrontamiento se encuentra la necesidad de información, apoyo y seguimiento que brindan las enfermeras/os ${ }^{(33,40-42)}$. Coincidiendo con esta afirmación, Charmaz ${ }^{(15)}$ añade además, la necesidad de información por parte de los profesionales sanitarios sobre alternativas a los problemas derivados de su propia enfermedad y tratamientos.

Es difícil llegar a un consenso donde se especifiquen las competencias que debería poseer la enfermera/o para cubrir las necesidades de los pacientes afectados por la EC, ya que son diversos los protocolos y guías creados a este respecto. Aun así la enfermera/o dedicada al cuidado de estos pacientes, debería ser capaz de desarrollar diferentes actividades, una vez adquirida la formación necesaria y adaptarlas a su propio contexto. Uno de los ejemplos más cercanos en el desarrollo de rol de la enfermera/o lo encontramos, en el Hospital Vall d'Hebron en Barcelona. El centro dispone de una Unidad de Atención Crohn-Colitis (UACC) para la atención integral a los pacientes con EC y colitis ulcerosa. La enfermera/o como miembro de un equipo multidisciplinar, realiza una gestión eficaz de atención a los pacientes con Ell, consiguiendo atender las necesidades de estas personas, sin aumentar el número de ingresos y acortando las estancias hospitalarias ${ }^{43,44)}$.

La enfermera/o puede intervenir en diferentes procesos de la enfermedad, desde el período en el que la enfermedad se encuentra en remisión, proporcionando la información necesaria para mejorar la calidad de vida, hasta los períodos de exacerbación, facilitando el acceso a tratamientos, pruebas o incluso ingresos hospitalarios ${ }^{(45)}$. Debe ser capaz de realizar un seguimiento de estos pacientes para ser conocedora en todo momento del estado en el que se encuentran las personas que tiene en su cartera de salud ${ }^{(2,46)}$. Esta cuestión, se muestra en diferentes estudios ${ }^{(47,48)}$ donde los autores concluyeron en la importancia del papel de la enfermería en el manejo de la enfermedad, proporcionando a los pacientes educación, asesoramiento y apoyo físico y emocional entre otros aspectos.

En cuanto a las competencias de enfermería según ANECA ${ }^{(49)}$, la enfermería trabajará con la cronicidad en general y con las personas afectadas de EC, de una manera holística, reconociendo e interpretando signos cambiantes de su salud (periodos de remisión y exacerbación), facilitando el contacto con otros especialistas. Así mismo, se describen competencias relacionadas con ser capaz de dar respuesta a las necesidades de los afectados ante situaciones similares a las que se presentan 
en esta patología, identificando estados de depresión, miedo, ansiedad, incertidumbre... y proporcionándoles apoyo y cuidados emocionales que permitan que los enfermos de EC expresen sus preocupaciones e intereses. Una vez analizados éstos, las enfermeras/os responderán adecuadamente para promover el bienestar del paciente.

Por último, y siguiendo el modelo de enfermería de D. Orem, en el que se expone que la enfermería es "ayudar al individuo a llevar a cabo y mantener por sí mismo acciones de autocuidado para conservar la salud y la vida, recuperarse de la enfermedad y/o afrontar las consecuencias de dicha enfermedad" la enfermera/o apoyará psicológicamente a la persona, promoverá un entorno favorable y educará a la persona y a su familia para recuperarse de la enfermedad y afrontar las consecuencias de la EC ${ }^{(48)}$.

\section{DISCUSIÓN DE DATOS}

El análisis de los datos derivado de la revisión de la literatura, muestra lo poco que se sabe acerca de la experiencia emocional de recibir el diagnóstico de EC y de las modificaciones y adaptaciones que tendrá que realizar la persona en su "nueva" vida.

Parece que nuestro sistema sanitario está pensado para la atención de patologías agudas y se resiste a cambiar su estructura, a pesar de que las enfermedades crónicas hayan aumentado de forma considerable en las últimas décadas.

La atención sanitaria, desde su visión medicalizada, se centra en el problema patológico en sí, obviando las luchas emocionales, sufrimiento o los factores sociales que intervienen en las experiencias de las personas con EC. Cuestión apoyada por el hecho de que en este contexto la mayoría de estudios acerca de la EC sean estudios cuantitativos con estadísticas y encuestas que no captan los relatos de los afectados por la EC ni sus mecanismos de afrontamiento para lidiar con la enfermedad.

Cabe mencionar que entre la literatura revisada, existen escasos estudios que sí tratan al enfermo crónico desde la perspectiva cualitativa, donde se pone de manifiesto las luchas y afrontamientos que deben realizar y los apoyos que buscan a través de diferentes redes sociales. En todas ellas la figura del personal sanitario (la mayoría hacen referencia a la figura del médico, obviando por completo al enfermero) aparece como una figura de referencia en la que las personas afectadas por la EC confían sus expectativas para volver a recuperar el control de sus vidas.

En cuanto a estudios concretos que muestren la relación y competencias de la enfermería con los enfermos de $\mathrm{EC}$, tampoco se ha recuperado abundante bibliografía ${ }^{(8,10,30,33,43,47,48)}$. Sin embargo, existen estudios donde se hace referencia a las competencias enfermeras para tratar con personas afectadas por esta patología, pero poniendo más énfasis en la realización de pruebas diagnósticas y aplicación de tratamientos ${ }^{(27,40,45)}$

En distintos artículos, se pueden agrupar algunas de las diferentes funciones que debería realizar una enfermera/o para proporcionar un cuidado integral a estos pacientes: poseer formación específica de los trastornos relacionados con el tracto digestivo, gestionar los signos y síntomas de la enfermedad, proporcionar toda la información necesaria acerca de los tratamientos instaurados, sus inconvenientes y la gestión de los diferentes efectos secundarios, prevenir complicaciones, controlar los efectos de la diarrea y el dolor, cuidados del estoma, consejos nutricionales, facilitar la 
accesibilidad al sistema formando parte de un equipo multidisciplinar, proporcionar apoyo psicológico y técnicas de afrontamiento ${ }^{(2,33,40,46-50)}$.

Resulta anecdótico comprobar cómo a pesar de que son muchas las ocasiones en las que las enfermeras/os se convierten en la única referencia de cómo manejar la EC, existen escasas publicaciones sobre este respecto. Este hecho podría estar relacionado con la medicalización de los sistemas sanitarios, quizá porque en un sistema medicalizado, como el nuestro, los sentimientos y emociones están silenciados por las propias enfermeras/os, ya que en una consulta de enfermería los enfermos de EC sólo informan sobre la sintomatología y adecuación de los tratamientos, pero en raros casos se les permite que expresen sus sentimientos y dificultades. Tendremos que reflexionar sobre por qué nos son invitados a expresar sus luchas emocionales.

\section{CONCLUSIÓN}

Las evidencias de esta revisión, muestran cómo la persona tras el diagnóstico de EC sufre una serie de cambios, no sólo a nivel físico, sino también psíquicos y sociales que requerirán del apoyo de un equipo de profesionales, entre ellos las enfermeras/os competentes para poder acompañar a las personas con patología de EC en su camino hacia la adaptación de su nueva condición de vida.

Esta revisión integradora ha hecho visible la necesidad de abrir nuevas líneas de investigación sobre el cuidado de la cronicidad en general y especialmente en EC. Rechazando los modelos biomédicos y centrándose en las personas enfermas que se ven forzadas a silenciar sus conflictos emocionales en un sistema que parece no darle la importancia que se merecen.

\section{BIBLIOGRAFÍA}

1. Heap GA, Van Heel DA. The genetics of chronic inflammatory diseases. Human Molecular Genetics 2009; (18): 101-106.

2. Michael WD. Fight back against inflammatory bowel disease. Nursing 2008; 38(11): 34-40.

3. Sicilia B, Vicente R, Gomollón F. Enfermedad de Crohn y colitis ulcerosa: discusión de la epidemiología clásica. Acta Gastroenterol Latinoam 2009; (39): 135145.

4. Moreira MC. Colitis ulcerosa \& Enfermedad de Crohn. Universidad de Barcelona 2009.

5. Casellas F, Panés J, García-Sánchez V, Ginard D, Gomollón F, Hinojosa J, et al. Costes médicos directos de la enfermedad de Crohn en España. PharmacoEconomics 2010; (1): 38-46.

6. Medina O. Encuentro con la cronicidad. Rev Desarrollo Cientif Enferm 2003; (11): 79-82.

7. Fernández JC, Simón MA, Bueno AM. Depresión, género y manifestaciones sistémicas en las enfermedades inflamatorias crónicas del intestino. Psicothema 2010; (22): 208-212.

8. Iglesias M, Barriero de Acosta M, Vázquez I, Figueiras A, Nieto L, Lorenzo A, et al. Impacto psicológico de la enfermedad de Crohn en pacientes en remisión: Riesgo de ansiedad y depresión. Rev Esp Enferm Dig 2009; (101): 249-257. 
9. Tobón S, Vinaccia S, Quiceno JM, Sandín B, Rojas ACN. Aspectos psicopatológicos en la enfermedad de Crohn y en la colitis ulcerosa. Avances en Psicología Latinoamericana 2007; (25): 83-95.

10. Sainsbury A, Heatley RV. Review article: Psychosocial factors in the quality of life of patients with inflammatory bowel disease. Alimentary Pharmacology and Therapeutics 2005;21(5):499-508.

11. Escudero M, García N, Prieto M, Pérez O, March J, López M. Fibromialgia: percepción de pacientes sobre su enfermedad y el sistema de salud. Estudio de investigación cualitativa. Reumatología Clínica 2010; (6): 16-22.

12. Shuman R. Vivir con una enfermedad crónica. Paidos Ibérica Ediciones SA 1999.

13. Ibarra X, Siles J. Rol del enfermo crónico: Una reflexión crítica desde la perspectiva de los cuidados enfermeros. Cultura de los Cuidados: Revista de enfermería y humanidades 2006; (20): 129-135.

14. Llorca G. La dignidad de vivir con la enfermedad. La cronicidad como destino. 2010.

15. Charmaz K. Loss of self: A fundamental form of suffering in the chronically ill. Sociology of Health and IIIness 1983; (5): 168-195.

16. Reza C, Castro M, Solano G, Torres D, Martínez V. El impacto del diagnóstico. Experiencia descrita por mujeres con hipertensión arterial. Esc Anna Nery Rev Enfer 2010; (14): 33-38.

17. Kübler-Ross E. Sobre la muerte y los moribundos. Grijalbo 2000.

18. Pont E. Visiones desde la enfermedad. Metas de Enfermería 2006; (9): 10-15.

19. Muñoz LA, Price Y, Gambini L, Stafanelli M. Significados simbólicos de los pacientes con enfermedades crónicas. Rev Esc Enferm USP 2003; (37): 77-84.

20. Anota R. Respuesta emocional del paciente ante el diagnóstico de diabetes Miellitus tipo 2. [Tesis Doctoral] Universidad Veracruzana 2008.

21. Bury M. Chronic illness as biographical disruption. Sociology of Health and IIIness 1982; (4): 167-182.

22. Barnett M. Chronic obstructive pulmonary disease: A phenomenological study of patient's experiences. Journal of Clinical Nursing 2005; (14): 805-812.

23. Faria APS, Bellato R. The everyday life of people living with the Chronic condition of diabetes Mellitus. Rev esc enferm USP 2009; (43): 752-759.

24. Mercado FJ, Ibarra E. Chronic illness from the perspective of patients and health professionals: A qualitative study in Mexico. Cadernos De Saúde Pública 2007; (23): 2178-2186.

25. Carlens C, Hergens MP, Grunewald J, Ekbom A, Eklund A, Hoglund CO, et al. Smoking, use of moist snuff, and risk of chronic inflammatory diseases. Crit. Care Med. 2010; (181): 1217-1222.

26. Suhal S, Kyle S, Roberto E, Carlton A, Susan MD. Smoking and inflammatory Bowel disease: A meta-analysis. Mayo Clinic Proceedings 2006; (81): 1462-1471.

27. Klement E, Cohen RV, Boxman J, Joseph A, Reif S. Breastfeeding and risk of inflammatory bowel disease: A systematic review with meta-analysis. The American Journal of Clinical Nutrition 2004; (80): 1342-1352.

28. Vega EG, Rodríguez CF. Algunos factores predictores en la enfermedad de Crohn. Psicothema 1998; (10): 143-151.

29. Diener JA. Everyone poops but no one wants to talk about it: The lived experiences of Young people with inflammatory Bowel disease. [Tesis Doctoral] Universidad de Canadá 2011.

30. Rodrigo L, Riestra S, Nino P, Cadahia V, Tojo R, Fuentes D, et al. A populationbased study on the incidence of inflammatory Bowel disease in Oviedo (northern Spain). Rev Esp Enferm Dig 2004; (96): 296-304. 
31. Camacho L. Factores psicosociales en la enfermedad crónica intestinal: estado actual. Rev. Ell al Día 2009; (8): 103-111.

32. Laura M, Dory P, Wallace V, Crandall MD. Review: Psuchosocial Issues in Pediatric Inflamatory Bowel Disease. Journal of Pediatric Psychology 2004; (4): 243-257.

33. Cònsul M, Soldevilla M. La enfermedad inflamatoria intestinal. Reflexiones sobre los cuidados enfermeros. Nursing 2004; (22): 58-64.

34. Lynch T, Spence D. A qualitative study of youth living with crohn disease. Gastroenterology Nursing 2008;31(3):224-230.

35. Pihl-Lesnovska K, Hjortswang H, Ek A-, Frisman GH. Patients' perspective of factors influencing quality of life while living with Crohn disease. Gastroenterology Nursing 2010;33(1):37-44.

36. Martínez A. El etnógrafo discapacitado. Acciones e Investigaciones sociales 2002; (15): 57-70.

37. Mattos M, Maruyama SAT. The experience of a person with Chronic Kidney disease in hemodialysis. Rev Gaúcha Enferm 2010; (31): 428-434.

38. Fletcher PC, Schneider MA, Van Ravenswaay V, Leon Z. I am doing the best that i can!: Living with inflammatory bowel disease and/or irritable bowel syndrome (Part II). Clinical Nurse Specialist 2008;22(6):278-285.

39. Altschuler A, Collins B, Lewis JD, Velayos F, Allison JE, Hutfless S, et al. Gastroenterologists' attitudes and self-reported practices regarding inflammatory bowel disease. Inflamm Bowel Dis 2008;14(7):992-999.

40. Woods $L$, Belling R, McLaren S. A systematic review of the effectiveness of inflammatory bowel disease specialist nurse. London South Bank University 2006.

41. Hall NJ, Rubin GP, Dougall A, Hungin A, Neely J. The fight for "health-related normality': a qualitative study of the experiences of individuals living with established inflammatory bowel disease (IBD). Journal of health psychology 2005;10(3):443-455.

42. Soares Sarlo R, Ribeiro Barreto C, Arena Moreira T. Understanding the experience of patients who have Crohn's Disease. Acta Paul Enferm 2008;21(4):629-35.

43. Casellas F, Borruel N, Torrejón A, Castells I. Efecto de la aplicación de un método de asistencia continuada centrada en el paciente con enfermedad inflamatoria intestinal en la actividad hospitalaria. Rev Esp Enferm Dig 2012; (104): 16-20.

44. Husain A, Triadafilopoulos $\mathrm{G}$. Communicating with patients with inflammatory bowel disease. Inflamm Bowel Dis 2004;10(4):444-450.

45. Cooper JM, Cillier J, James V, Hawkey JC. Beliefs about personal control and self-management in 30-40 year olds living with inflammatory bowel disease: $\mathrm{A}$ qualitative study. Int J Nurs Stud 2010; (47): 1500-1509.

46. Stansfield C, Fraser A, Thompson J, Povey J. Roles descriptive for inflammatory bowel disease nurse specialists. Royal College of Nursing 2007; 1-20.

47. Hernández-Sanpelayo P, Seoane M, Oltra L, Marín L, Torrejón A, Vera MI, et al. Contribution of nurses to the quality of care in management of inflammatory Bowel disease: A synthesis of the evidence. Journal of Crohn's and Colitis 2010; (4): 611-622.

48. Belling R, Woods L, MacLaren S. Stakecholder perceptions of specialist Inflammatory Bowel Disease nurses's role and personal attributes. International Journal of Nursing Practice 2008; (14): 67-73.

49. ANECA. Libro Blanco para la enfermería $2^{\mathrm{a}}$ ed. Barcelona; 2005.

50. Orem D. Modelo de Orem: Conceptos de enfermería en la práctica. Ediciones científicas y técnicas. Barcelona; 1993. 
ISSN 1695-6141

(๖) COPYRIGHT Servicio de Publicaciones - Universidad de Murcia 